\section{Dizziness and prevention of falls in the elderly}

\author{
Augusto P. Casani, Elena Navari \\ Department of Medical and Surgical \\ Pathology, Otorhinolaryngology Unit, \\ Pisa University Hospital, Pisa, Italy
}

\begin{abstract}
Disequilibrium is one of the most common complaints that older adults bring to their physician and falling is a frequent complication; because of the increasing number of elderly patients in western society the problem grows. The increasing susceptibility to falling can be consequence of age-related physiological changes and of a higher prevalence of comorbidities causing dizziness, vertigo and imbalance; these symptoms can worse the already poor balance of old adults increasing the risk of falling. Among the major reasons of vertigo and dizziness, central vertigo and orthostatic vertigo are very common; also vestibular disorders have a great role and are currently thought to account for $48 \%$ of dizziness reported by older adults. An early identification of the treatable condition underlying dizziness in elderly would surely ameliorate the outcome of these patients.
\end{abstract}

\section{Report}

Elderly are increasing over the last decades and falling is a frequent complication. It not only causes medical and social impairments, such as fall-related injuries and loss of independence, but it also induces fear of falling, which can result a substantial decline in quality of life. ${ }^{1}$ Consequently, accidental falls in elderly are one of the most important socio-health care problems of ageing western societies and dizziness is one of the major factors increasing the risk of falling. ${ }^{2}$

It is known that our balance is achieved and maintained by a complex set of sensorimotor control systems that include sensory input from vision, proprioception, and the vestibular system, the integration of these sensory inputs, and motor output to the eye and body muscles [vestibulo-ocular reflex (VOR), vestibulo-spinal reflex (VSR)]. Cerebral cortex and cerebellum contribute with learned information and coordination.

In elderly all the subsystems involved in balance control worsens. Decreasing in peripheral and central sensory function (visual, vestibular, somatosensory), muscles impairment (sarcopenia, arthritis), and cognitive and mental disorders (dementia, anxiety) are widely present in aging and causes static and dynamic postural impairment. Because a poorer postural control increases the risk of falling, it is well described in literature a relationship between gait speed and survival. ${ }^{3}$

Dizziness, vertigo, and imbalance represent common complaints that old adults bring to their physician; they are not normal part of aging and worsen the already poor equilibrium of elderly. Since dizziness is a major contributing factor to falls and it is described that adult dizzy patients have an increased mortality rate compared to not dizzy adults, ${ }^{4}$ the early identification and treatment of its causes is mandatory.

In our experience, according with Jahn et al.,$^{5}$ central and orthostatic vertigo are very common, but also peripheral vestibular disorders have a great role; it is currently thought that vestibulopathies account for $48 \%$ of dizziness reported by older adults. ${ }^{6}$ In Table 1 the possible etiologies of dizziness and vertigo in the elderly are presented. Benign paroxysmal positional vertigo (BPPV), unilateral and bilateral vestibular failure (UVF and BVF, respectively), Ménière's disease (MD) are very common disorders, able to afflict the balance and increase the risk of falling; in the most of cases a early identification and treatment of these conditions greatly improves the balance of patient and their outcome.

In order to correctly manage an old adult complaining dizziness or vertigo the physician needs to carefully investigate about the history, paying special attention to medical therapies, examine the standing posture and gait, the sensory and musculoskeletal system, and cognitive function. Questionnaire as the dizziness handicap inventory (DHI) can be helpful in order to estimate the degree of the disequilibrium.

BPPV represents the most frequently reported vestibular disorder in neurootological clinical practice and is the most frequent cause of vertigo with a prevalence in the general population of about $2.4 \%{ }^{7}$ This disorder is characterized by the recurrence of brief and violent crises of true vertigo (spinning dizziness) triggered by horizontal and vertical movements of the head. Each episode of vertigo has an abrupt and sudden onset that lasts for a short time (few seconds to 1 minute) and tends to disappear rapidly.

The pathogenesis of BPPV may be identified in the detachment of otoliths (calcium crystals that physiologically participate in the constitution of the otolithic membrane of the utricle and the saccule), and in their displacement in one of the three
Correspondence: Augusto P. Casani, Department of Medical and Surgica Pathology, Otorhinolaryngology Section, Pisa University Hospital, via Paradisa 2, 56126 Pisa, Italy.

E-mail: augusto.casani@unipi.it

Key words: Dizziness; vertigo; elderly; vestibular; falls.

Contributions: APC, conception and design of the report, evaluation of patients, drafting the article, critical revision. EN, contribution to the report design, evaluation of patients, drafting the article, critical revision.

Conflict of interest: the authors declare no potential conflict of interest.

Conference presentation: paper presented at $31^{\circ}$ Congresso Nazionale SIGOT - Genova, June 8-9, 2017.

Received for publication: 25 October 2017. Accepted for publication: 9 November 2017.

This work is licensed under a Creative Commons Attribution-NonCommercial 4.0 International License (CC BY-NC 4.0).

(C) Copyright A.P. Casani and E. Navari, 2017 Licensee PAGEPress, Italy

Geriatric Care 2017; 3:7160

doi:10.4081/gc.2017.7160

semicircular canals which, ordinarily sensitive only to angular acceleration, acquires the ability to perceive also changes of position in relation to the axis of gravity. It is also possible that particles differing from the otoconia (clots, white blood cells, etc.) may be responsible for the disorder.

The etiology of BPPV has still not been fully understood and is classified as idiopathic in more than $70 \%$ of cases. In the remaining cases, its onset may be associated with other events that involve the labyrinth such as a viral labyrinthitis, a sudden loss of the unilateral vestibular function due to vascular causes or surgical interventions of an otological nature. In order to diagnose BPPV, a careful anamnesis and the clinical examination of the patient using specific diagnostic maneuvers according to which of the canals is involved are needed. In order to obtain remission of the symptomatology some maneuvers are used again performed on the same plane of the involved canal with the aim of inducing the repositioning of the ectopic material in the vestibule.

While this disorder may actually arise at any time of life, it manifests more frequently in adults and elderly people with a peak of incidence around the $5^{\text {th }}-7^{\text {th }}$ decade of life, maybe because of the higher probability of 
detachment of otoconia with age. A correct diagnostic approach to the disorder means avoiding a series of useless and costly instrumental tests and inappropriate treatment and enables clinicians to go ahead swiftly with the treatment. In many cases, patients affected by BPPV suffer the effects of late diagnoses and the consequences of starting late with the therapy in the range of several months. The importance of timely recognition of BPPV derives also from the high incidence of this pathology in the elderly population, where the presence of this disorder inevitably leads to a worsening of the capacity to carry out normal routine daily activities, determining moreover a significant increase in risk of falling, and consequent injury. In the light of this eventuality, it is clear that a more timely identification and treatment of this disorder would have positive repercussions on the quality of life of the patient, particularly in the elderly subject, as well as containing the costs incurred by the health system and by society.

After the therapeutic maneuvers have been performed, also in the case of a successful outcome, up to two-thirds of patients may perceive a prolonged sensation of instability, also known as residual dizziness. This residual symptomatology, which in part may be attributed to a state of otolithic dysfunction, would appear to be correlated with the duration of the illness, the old age and the anxiety. In the light of this observation, again early recognition and treatment of a clinical picture of BPPV would allow clinicians to significantly reduce the incidence of this troublesome disorder

UVF is a frequent condition characterized by an asymmetrical vestibular functioning due to a diminished sensory input of one vestibular side. The etiology is unknown but evidence suggests that the damage is often caused by reactivation of a latent Herpes simplex virus type 1 infection; in the other cases a vascular damage, such as in a condition of vertebrobasilar insufficiency, extremely frequent in elderly, can be speculate.

The sudden unilateral loss or impairment of the vestibular function causes vertigo, dizziness and impaired postural function. After the acute phase of the disease, most of patients recovery the disability because of the plasticity of the central nervous system; however, until to $50 \%$ of patients develop chronic either continuous or paroxysmal dizziness after UVF. Among the factors affecting the recovery, surely the old age represents the more significant.

BVF is a condition frequently overlooked in older patients. Among the symptoms, chronic dizziness and oscillopsia are prevalent and due to the bilateral impairment of the peripheral vestibular function that compromise the VSR and VOR. In addition to the clinical history, the bilateral VOR deficit will confirm the diagnosis. In about $50 \%$ of cases no specific cause is

Table 1. Possible etiology of dizziness in the elderly.

\begin{tabular}{ll}
\hline Peripheral vestibular & Benign paroxysmal positional vertigo \\
& Unilateral vestibular failure \\
& - Vestibular neuritis \\
& - Occlusion of the anterior vestibular artery \\
& Bilateral vestibular failure \\
& Let onset Ménière's disease or Tumarkin's otholitic crisis \\
& Transient ischemic attack of vertebrobasilar artery \\
Central nervous system & Neurodegenerative disorders \\
& Downbeat and upbeat nystagmus syndromes \\
\hline Cardiovascular & Arrhythmias \\
& Postural hypotension \\
& Congestive heart failure \\
Heart valve failure \\
Blood pressure imbalance
\end{tabular}

\begin{tabular}{ll} 
Medications & Antihypertensive \\
& Benzodiazepines \\
& Hypnotics \\
& Anxiolytics \\
& Antiepileptic \\
\hline Multimodal balance disorder & Presbyastasis \\
Visual defects & Vision loss \\
& Cataracts \\
\hline Others & Musculoskeletal system disorders (osteoarthritis, etc.) \\
\hline
\end{tabular}

identified when a diagnosis is made; in the remaining cases, ototoxic medications or pre-existing inner ear disorders are often implicated.

MD is an idiopathic inner ear disorder characterized by spinning dizziness (lasting from $20 \mathrm{~min}$ to $24 \mathrm{~h}$ ), fluctuating hearing loss, ear fullness, and tinnitus. The natural history of the disease is characterized by variable periods of exacerbation and remission of symptoms. The diagnosis is clinical. Vertigo spells can be controlled in the most of patients by using labyrinth-sparing therapies; if it is not, an ablative approach with low-dose intra-tympanic gentamicin (ITG) has proved to assure a very good control on the vertigo spells.

Some patients affected by MD, especially if old and in the later stage of the disease, can complain Tumarkin's otolithic crisis, drop attacks in which the person falls to the ground with no warning, remains awake and does not lose consciousness. Also in this case a therapy with ITG can be useful. The major side effect of this treatment is the unsteadiness, due to the drug damage to the vestibular labyrinth.

In some of the above conditions (residual dizziness post-BPPV, chronic dizziness after UVF or BVF, unsteadiness after ITG, etc.) a vestibular rehabilitation program represents an important element of treatment with active gait training and postural and oculomotor stabilization training. Recent reviews in literature describe the effectiveness of this approach in vestibular patients by improving the VOR and VSR function; ${ }^{8}$ the treatment would decrease the fall risk improving the quality of life of the patients.

A lot of central vestibular disorders can be responsible for chronic dizziness or recurrent vertigo in older adults, because of lesions affecting structure and pathway involved in the oculomotor control and postural regulation. Strokes, brain tumors represent frequent conditions. The clinical findings and further investigations using imaging will confirm the suspicion. Among the central causes of dizziness in elderly, white matter lesions (WML) has generated great interest over the last years. Hamad et $a l .{ }^{9}$ report that the severity of WML is higher in elderly patients with no specific cause for their dizziness; therefore, the authors postulate that WML may induce dizziness and increased fall risk either because patients perceive a degree of objective unsteadiness or by a cortical-subcortical disconnection syndrome, secondary to disruption of white matter tracts involved in gait and balance control. Elderly patients with dizziness, without a clear diagnosis and despite clinical and vestibular assessment, should undergo brain imaging to 
assess the level of WML. On the other hand, clinicians should be cautious when ascribing dizziness solely to the presence of mild or moderate WML; given the high incidence of vestibular disease in neurovascular or geriatric clinics, easily treatable peripheral vestibular causes may be missed..$^{10}$

The current treatment for WML dizziness involves preventive measures including control of vascular comorbidities; customized retraining through a combination of rehabilitation and possibly neuromodulation of cortical pathways may form part of future potential treatment options for this subgroup of dizzy patients. ${ }^{9}$

The association of Citicoline, Melissa officinalis, Ginger and Vinitrox appears helpful in improving the dizziness not related to a define pathology in elderly. In a study, designed as prospective and randomized case-control, is reported a statistically significant improvement of the DHI score and of the length and surface values measured with posturography after 60 days in patients taking the nutraceutic tablet compared to the patient who did not. ${ }^{11}$ The same association, in a previous study, showed positive effects on residual dizziness after UVF and BPPV. ${ }^{12}$

In conclusion, vertigo and dizziness are frequent conditions among elderly. They can lead to disequilibrium, fear of falling, inactivity, and increased fall risk. Since in some cases the underlying causes can be treated or improved, the clinician should take better care of these conditions, not just ascribing them to the aging.

\section{References}

1. World Health Organization (WHO). WHO global report on fall prevention in older age. Geneva: World Health Organization; 2007.

2. Ciorba A. Dizziness and the risk of falling in the elderly: a literature review. J Hear Sci 2015;5:9-13.

3. Studenski S, Perera S, Patel K, et al. Gait speed and survival in older adults. JAMA 2011;305:50-8.

4. Corrales CE, Bhattacharyya N. Dizziness and death: an imbalance in mortality. Laryngoscope 2016;126:2134-6.

5. Jahn K, Kressig RW, Bridenbaugh SA, et al. Dizziness and unstable gait in old age: etiology, diagnosis and treatment. Dtsch Arztebl Int 2015;112:387-93.

6. Herdman SJ. Vestibular Rehabilitation. 3rd ed. Philadelphia: F.A. Davis Company: 2007.
7. Von Brevern M, Radtke A, Lezius F, et al. Epidemiology of benign paroxysmal positional vertigo: a population based study. J Neurol Neurosurg Psychiatry 2007;78:710-5.

8. McDonnell MN, Hillier SL. Vestibular rehabilitation for unilateral peripheral vestibular dysfunction. Cochrane Database Syst Rev 2015;1:CD005397.

9. Ahmad H, Cerchiai N, Mancuso M, et al. Are white matter abnormalities associated with "unexplained dizziness"? J Neurol Sci 2015;358:428-31.

10. Cerchiai N, Mancuso M, Navari E, et al. Aging with cerebral small vessel disease and dizziness: the importance of undiagnosed peripheral vestibular disorders. Front Neurol 2017;8:241.

11. Casani AP, Caranzano F, Cerchiai N, et al. Valutazione sperimentale controllata di un preparato nutraceutico nel trattamento della "dizziness cronica". Acta Otorhinolaryngol Italica 2017;11:1-9.

12. Benazzo M, Cerchiai N, Comacchio F, et al. Valutazione sperimentale controllata di un nuovo preparato nutraceutico nel trattamento della fase post-critica del deficit evstibolare acuto e della vertigine parossistica posizionale benigna. Acta Otorhinolaryngol Italica 2016; 10:1-10. 